\title{
A QoS-Aware CAC with Bandwidth Reservation and Degradation Scheme in IEEE 802.16e Networks
}

\begin{abstract}
Call admission control (CAC) schemes play a critical role in providing quality-of-service (QoS) guarantees for various classes of traffic with diverse QoS requirements in IEEE 802.16e networks. The dynamic CAC and bandwidth reservation scheme is one of the current schemes that simultaneously provides efficient utilization of network resources and guarantees QoS for admitted connections. However, its admission criteria starved high and low service classes due to its linear adaptation policy to accommodate more users into the network under moderate-to-heavy traffic load conditions. Its adaptive threshold for handoff connections is adjusted based on the arrival of new and handoff connections, that results in a waste of resources when the new and handoff connection arrival rate occurs frequently. In this paper, a new CAC scheme for Mobile WiMAX networks is proposed to prevent starvation of service classes and enhance the efficient utilization of network resources. The scheme determines a new admission criteria based on a scheduling service class. In the admission criteria, bandwidth degradation policy is used to admit more users when there is no available bandwidth to admit a more users. The adaptive threshold has been introduced dynamically to adjust the quantity of reserved bandwidth for handoff connections based on the traffic intensity of handoff requests. In addition, an analytical model for the proposed scheme is also developed. Extensive simulation experiments have been conducted to evaluate the performance of our proposed approach. The simulation results illustrate that the proposed scheme significantly improves the network efficiency compared to other schemes in terms of accepting more connections into the network and assuring QoS for all service classes. The numerical results have shown similar performance to the simulation results.
\end{abstract}

Keyword: Mobile WiMAX; Handoff connection; New connection; Admission criteria; Adaptive threshold 